\title{
Evolution de l'impact des retombées de Tchernobyl sur les poissons de rivières, de retenues artificielles et de lacs de montagne corses ${ }^{*}$
}

\author{
B. DESCAMPS **
}

(Manuscrit reçu le 3 août 1990)

\begin{abstract}
RÉSUMÉ Cet article est relatif à l'évolution de l'impact des retombées de Tchernobyl sur les poissons de Corse pendant trois ans : juillet 1986-juillet 1989. Les principales catégories d'eaux de surface ont été étudiées : les six principales rivières, deux retenues artificielles destinées à l'irrigation et six lacs d'altitude.

On compare les réponses locales des différentes espèces constituant les peuplements dominants avec celles d'autres rivières françaises et européennes. L'impact sanitaire potentiel est calculé.

ABSTRACT The trend of the impact of the Chernobyl accident fallout on fish in Corsica was followed during three years, from July 1986 to July 1989. The main categories of surface water were studied : the chief six rivers, two artificial irrigation dams and six altitude lakes. The local responses of several species making up the prevailing stock were compared with those of other french or european rivers. the potential health impact was assessed.
\end{abstract}

\section{INTRODUCTION}

Dès les premiers jours de mai 1986 on a su qu'un panache radioactif issu de l'accident de. Tchernobyl avait survolé, en particulier, la partie de la Méditerranée où se trouve la Corse. La consommation de poissons étant l'une des voies qui mènent directement à l'homme, on s'est plus particulièrement intéressé à ce maillon important dans des lacs de haute montagne, des retenues artificielles servant à l'irrigation et des fleuves de la côte orientale mais aussi de la côte occidentale. Le but de cet article est d'abord de donner les concentrations enregistrées pour les trois principaux radionucléides décelés, à la même époque - juillet - en 1986,

* Ce travail est essentiellement réalisé au sein du Laboratoire de Radioécologie des Eaux Continentales du Centre d'Etudes de Cadarache, BP 1, 13108 Saint-Paul-Lez-Durance Cedex.

** Commissariat à l'énergie atomique, Institut de Protection et de sûreté nucléaire (IPSN), Service d'Etudes et de recherches sur l'environnement (SERE), Laboratoire de radioécologie du bassin rhodanien, CEN Valrho, site de Pierrelatte, BP 111, La Blachette, 26700 Pierrelatte. 
1987, 1988 et 1989. Par ordre décroissant d'importance, il s'agit de ${ }^{137} \mathrm{Cs},{ }^{134} \mathrm{Cs}$ et ${ }^{11.0 \mathrm{~m}} \mathrm{Ag}$. Citons la présence anecdotique, mais cependant significative, de ${ }^{103} \mathrm{Ru}$ et de ${ }^{106} \mathrm{Ru}+\mathrm{Rh}$ en juillet 1986 en un lieu.

Le cas de la Corse devant être replacé dans un contexte européen, nous avons ensuite comparé ces valeurs à celles enregistrées en particulier dans le nord de l'Italie, à peu de distance, mais aussi dans l'Europe du Nord et plus particulièrement en Scandinavie, là où on a enregistré les concentrations les plus importantes.

L'aspect sanitaire n'étant pas à négliger, surtout dans cette région, nous avons mis en évidence l'évolution de la dose induite par la consommation annuelle de $10 \mathrm{~kg}$ de poissons, et ceci pour différentes espèces et différents lieux.

\section{RAPPELS SUR L'ACCIDENT}

Le début de l'accident date du 26 avril 1986 et les rejets de produits radioactifs dans l'atmosphère ont duré, pour l'essentiel, 10 jours (jusqu'au 5 mai). Le terme source est estimé au maximum à 200 millions de curies $\left(7,4.10^{6} \mathrm{TBq}\right)$ soit environ $10 \%$ de l'inventaire du cœur au moment de l'explosion. Les gaz rares ont été rejetés à $10 \%$ et les produits volatils (I, Cs, Te) entre 10 et $20 \%$ [1].

Le premier paramètre à intervenir à la suite de l'accident est constitué par l'ensemble des conditions météorologiques locales durant les deux premiers jours (26 et 27 avril). Ces conditions météorologiques locales, et en particulier la présence et la localisation des zones d'inversion de température, ont influencé, en particulier, la hauteur de migration verticale des différents panaches de départ. Pour ce qui concerne la zone que nous avons étudiée, il semble que les rejets effectués le 27 avril vers 12 heures, à $1500 \mathrm{~m}$ de haut, soient à l'origine des retombées de Corse. L'heure d'émission des rejets est très importante puisque ceux effectués, le même jour, vers $0 \mathrm{~h}$ ont touché l'Europe de l'est, le nord de l'Italie, le Bénélux, le Danemark et l'est de la France alors que ceux effectués à partir de $18 \mathrm{~h}$ ont touché la Grèce. Les émissions du 26 ont, pour leur part, atteint essentiellement la Scandinavie (Suède et Finlande) et celles du 28 l'est de l'URSS, la Mer Noire, la Turquie et le Proche-Orient. L'Italie du sud et la Grèce sont, une nouvelle fois, atteintes par des rejets émis le 29 [1].

\section{CARACTÉRISTIQUES DES FLEUVES, LACS ET RETENUES ÉTUDIÉS}

Les dimensions réduites de la Corse et la présence d'une dorsale montagneuse importante conduisent les eaux de surface à former des rivières à pente importante et à petits bassins versants. Les profils en long de ces rivières présentent deux tronçons distincts : le premier, sur la 
majeure partie du cours, avec une pente longitudinale forte associée à une vallée encaissée tandis que le second voit la pente passer très rapidement à moins de 0,3 \% entraînant la formation d'une plaine triangulaire.

Nous avons étudié les six rivières les plus importantes; il s'agit, du nord au sud :

- sur la façade orientale, Le Golo, le Tavignano, le Fium'Orbo ;

- sur la façade occidentale, Le Liamone-Cruzzini, le Gravone et le Taravo.

Elles sont caractérisées par une faible longueur (entre $80 \mathrm{~km}$ pour le Tavignano et $44 \mathrm{~km}$ pour la Gravone), une altitude maximale élevée (entre $2710 \mathrm{~m}$ pour le Golo et $2042 \mathrm{~m}$ pour le Taravo) et, corrélativement, par une pente moyenne importante (entre 5,3\% pour la Gravone et le Liamone-Cruzzini et 3,2 \% pour le Taravo) leur conférant un caractère torrentiel marqué. Leur débit moyen annuel est assez faible puisque compris entre 4 et $17,3 \mathrm{~m}^{3} \cdot \mathrm{s}^{-1}$ (tableau I).

Huit étendues d'eau ont aussi été inventoriées :

- six lacs de montagne, d'origine glaciaire, Nino, Capitello, Melo, Rotondo, Bracca, Vitalaca ; Vecchio,

- une retenue de moyenne montagne, l'Ospedale, près de Porto

- une retenue collinaire de la plaine orientale, Teppe Rosse, près d'Aléria.

Le tableau II indique, pour chacune d'elles, le massif montagneux concerné, l'altitude, la rivière dont elle est l'origine (si c'est le cas), les dimensions (longueur et largeur, etc.).

TABLEAU

Caractéristiques des fleuves étudiés

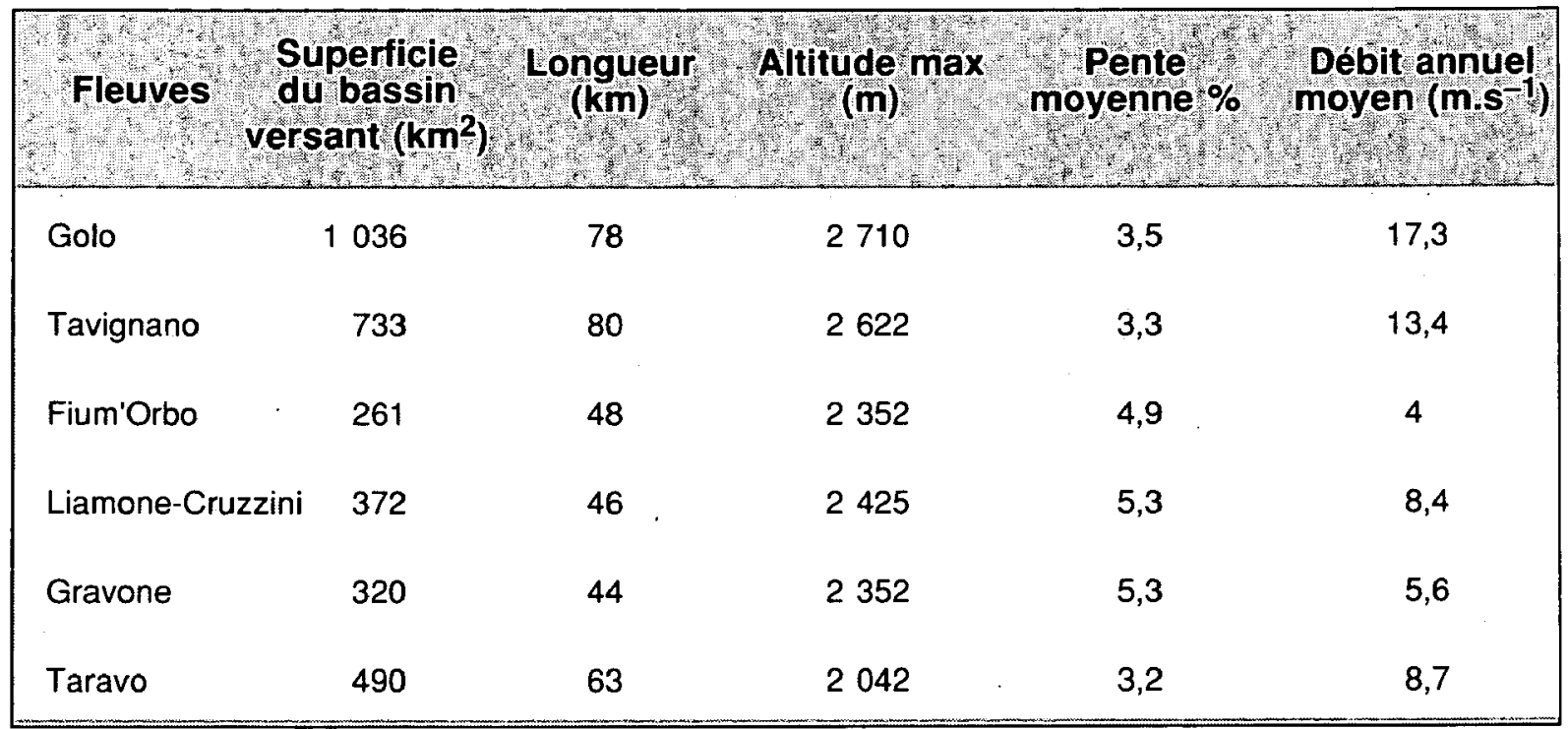


TABLEAU II

Caractéristiques des lacs de haute montagne et des retenues de l'Ospedale et de Teppe Rosse

\begin{tabular}{|c|c|c|c|c|c|c|c|c|}
\hline $\begin{array}{l}\text { Lacs } \\
\text { retenues }\end{array}$ & $\begin{array}{c}\text { Massif } \\
\text { concerne }\end{array}$ & $\begin{array}{c}\text { Altitude } \\
\text { (m) }\end{array}$ & Source de & $\begin{array}{l}L \times 1 \text { SL } \\
\text { (m) }\end{array}$ & $\begin{array}{c}\text { Superficie } \\
\text { ha) }\end{array}$ & $\begin{array}{c}\text { Perimètre } \\
\text { (m) }\end{array}$ & $\begin{array}{c}\text { Profondeur } \\
\text { (m) }\end{array}$ & $\begin{array}{c}\text { Superficie } \\
\text { bassin } \\
\text { versant } \\
\text { (ha) }\end{array}$ \\
\hline Nino & Rotondo & 1743 & Tavignaro & $400 \times 226$ & 6,5 & 1000 & 11 & 104 \\
\hline Capitello & Rotondo & 1930 & $\rightarrow$ Melo & $325 \times 250$ & 5,5 & 916 & 42 & 47 \\
\hline Melo & Rotondo & 1711 & Restonica & $300^{1}$ & 6,2 & 930 & 15,5 & 173 \\
\hline Rotondo & Rotondo & 2231 & Rotondo & $425 \times 225$ & $5 \quad 7,4$ & 1200 & 35 & 50,4 \\
\hline Bracca & Renoso & 2085 & & $120 \times 100$ & 0,8 & 450 & 6,5 & 11 \\
\hline Vitalaca & Renoso & 1777 & Prunelli & $175 \times 60$ & 0,6 & 400 & 3,5 & 128 \\
\hline \multicolumn{2}{|l|}{ Ospedale } & 949 & Palavesavi & $1600 \times 200$ & 0445 & 4000 & 20 & 250 \\
\hline \multicolumn{2}{|c|}{ Teppe Rosse } & 30 & vallée sèche & $1400 \times 450$ & $50 \quad 55$ & 3800 & 11 & 350 \\
\hline
\end{tabular}

1. Forme circulaire, diamètre $=300 \mathrm{~m}$

Les campagnes de pêche ont toutes été faites durant l'été, le plus souvent durant la première décade de juillet, des années 1986, 1987, 1988 et 1989. Deux techniques ont été utilisées :

- la pêche électrique a été pratiquée dans les rivières et à pied, compte tenu de leur configuration (elle peut être réalisée en bateau sur les grandes rivières, les étangs et les lacs peu profonds) ;

- la pêche aux filets a été mise en œuvre dans les deux retenues de l'Ospedale et de Teppe Rosse et dans les six lacs de montagne.

Le matériel de pêche utilisé a été aimablement prêté par $M$. Roche du Service régional d'aménagement des eaux (SRAE) de Haute Corse. Les pêches proprement dites ont été effectuées sous la responsabilité et par le Conseil supérieur de la pêche (CSP) de ce département et, plus précisément, par ses trois garde-pêches. Pour les lacs de haute montagne, il y a eu collaboration entre le SRAE, le CSP, le CEA, mais aussi le Parc national régional corse (PNRC). Ces pêches en lacs ont également servi de support à des études strictement hydrobiologiques (inventaire et évolution des peuplements aquatiques), à l'initiative du Centre technique du machinisme agricole, du génie rural, des eaux et des forêts (CEMAGREF) d'Aix-en-Provence [6]. Le matériel nécessaire à ces études écologiques en lacs de haute montagne a nécessité un héliportage autorisé par le PNRC. 
La première campagne de prélèvements de juillet 1986 a dû être organisée très rapidement, aussi les pêches n'ont-elles pu être réalisées que dans le Tavignano (zones amont et aval), le Fium'Orbo (zone aval) et la retenue de Teppe Rosse. En 1987, à ces mêmes lieux, nous avons ajouté les zones amont et aval du Golo et les lacs de Nino et de Rotondo. En 1988, nous nous sommes aussi intéressés à la retenue de l'Ospelade, aux trois rivières de la côte occidentale (Liamone-Cruzzini, Gravone et Taravo), aux lacs Melo et Capitello et une nouvelle fois, au lac Nino. En 1989, la campagne la plus intense a vu un effort important, quand cela était possible, pour le prélèvement de différentes grosseurs d'individus. Cet effort de pêche devait être réalisé au moins une fois afin de connaître les réponses des différents classes d'âge des poissons ; on devait donc, de cette manière, tester la "mémoire" des poissons vis-à-vis des retombées de l'accident. Globalement, toutes les rivières et zones déjà citées ont été échantillonnées en 1989 ; pour les lacs, notre connaissance s'est complétée en s'intéressant aux lacs de Bracca et Vitalaca (fig. 1).

Les tableaux III pour les rivières et IV pour les retenues et les lacs récapitulent tous les prélèvements effectués et le poids moyen des individus. En rivière, les deux espèces dominantes sont l'anguille (Anguilla anguilla) et la truite (Salmo trutta fario), la tanche (Tinca tinca) et le muge (Mugil sp.) n'ayant été prélevés qu'une fois dans la zone aval du Tavignano. Dans les lacs de haute montagne, on enregistre la présence de la truite déjà citée et du saumon de fontaine (Salvelinus fontinalis). Dans le Teppe Rosse, on a pu inventorier cinq espèces, dont deux carnassières : le sandre (Lucioperca lucioperca) et la perche commune (Perca fluviatilis). Les trois autres espèces sont la tanche déjà citée, la carpe commune sous ses deux aspects "miroir" et "écailleuse" (Cyprinus carpio) et le rotengle (Scardinius erythrophthalmus). Pour la retenue de l'Ospedale, en plus de la tanche, du rotengle, de la perche et de la carpe "miroir", on a aussi prélevé le carassin (Carassius auratus) sous des formes plus ou moins hybrides.

\section{RÉSULTATS}

\section{Concentrations obtenues pour les poissons de Corse}

L'influence des retombées de Tchernobyl sur les poissons de Corse se caractérise essentiellement par la présence, dès juillet 1986 et jusqu'en juillet 1989 , date de notre dernière campagne de prélèvements, de ${ }^{137} \mathrm{Cs}$ et ${ }^{134} \mathrm{Cs}$. Les données sur ces deux radionucléides constituent donc l'essentiel de nos résultats, mais il faut aussi y ajouter quelques valeurs relatives à l'argent $110 \mathrm{~m}$ et, encore plus rarement, à ${ }^{103} \mathrm{Ru}$ et à ${ }^{106} \mathrm{Ru}+\mathrm{Rh}$. Nous fournirons aussi dans ce chapitre les valeurs obtenues sur les unios lamellibranches d'eau douce autochtones de certaines rivières corses prélevés en 1986, 1987, 1988 et 1989 à la station Tavignano aval. En effet, ces lamellibranches sont généralement considérés comme d'assez bons indicateurs de la pollution métallique [5], donc de la pollution radiologique. 


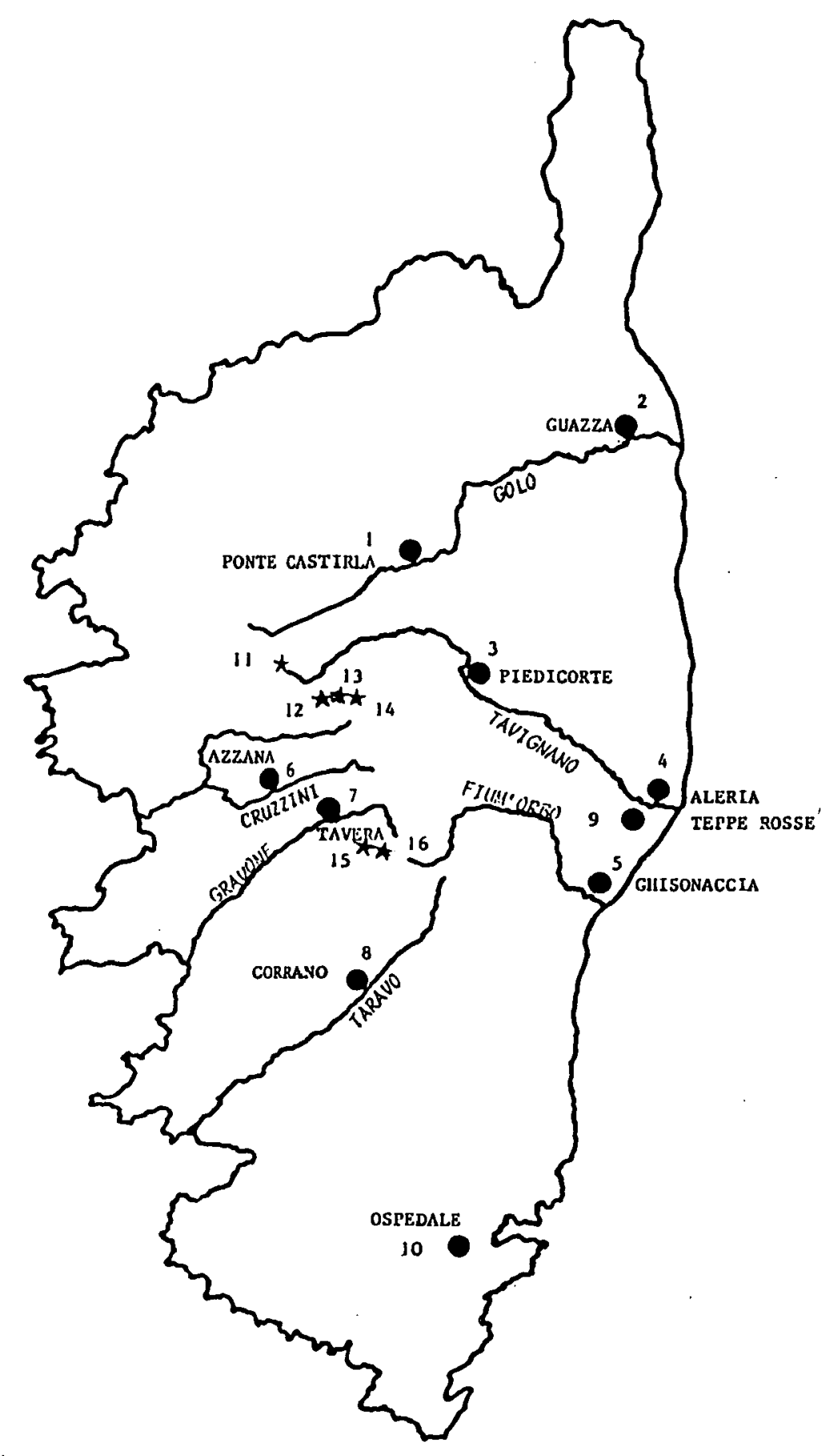

1 Ponte Castirla, amont Golo 5 Ghisonaccia, aval Fium'Orbo

9 Teppe Rosse 13 Lac Capitello 2 Guazza, aval Golo 6 Azzana, amont Liamone-Cruzzini 10 Ospedale 14 Lac Rotondo 3 Piedicorte, amont Tavignano 7 Tavera, amont Gravone 11 Lac Nino 15 Lac Bracca 4 Aléria, aval Tavignano

8 Corrano, amont Taravo 12 Lac Melo 16 Lac Vitalaca

Fig. 1 - Lieux de pêches dans les rivières, retenues et lacs corses de 1986 à 1989 
TABLEAU III

Espèces prélevées dans les rivières corses en juillet 1986, 1987, 1988 et 1989 Poids moyen des lots mesures $(g)$.

$A$ anguille, $M$ muge, $T$ truite, TA Tanche

\begin{tabular}{|c|c|c|c|c|c|c|c|c|c|c|}
\hline \multirow{3}{*}{$\begin{array}{l}\text { Rivières } \\
\text { Golo }\end{array}$} & \multirow{3}{*}{ Zones } & \multirow{3}{*}{\begin{tabular}{l}
\multicolumn{1}{c}{ Lieux } \\
Ponte Castirla \\
Viru $^{1}$
\end{tabular}} & \multicolumn{2}{|c|}{1986} & \multicolumn{2}{|c|}{1987} & \multicolumn{2}{|c|}{1988} & \multicolumn{2}{|c|}{1989} \\
\hline & & & & & T106 & $\begin{array}{l}\text { A } 140 \\
\text { A296 }\end{array}$ & $\begin{array}{l}\text { T42 } \\
\text { T110 }\end{array}$ & A209 & $\begin{array}{l}\text { T57 } \\
\text { T139 }\end{array}$ & $\begin{array}{l}\text { A65 } \\
\text { A267 }\end{array}$ \\
\hline & & & & & & 2 & $\begin{array}{l}\text { T25 } \\
\text { T70 }\end{array}$ & , & $\begin{array}{l}\text { T25 } \\
\text { T70 }\end{array}$ & \\
\hline & Aval & Guazza & & & & & T134 & $\begin{array}{l}\text { A58 } \\
\text { A84 }\end{array}$ & T133 & $\begin{array}{l}\text { A20 } \\
\text { A 40 } \\
\text { A65 } \\
\text { A267 }\end{array}$ \\
\hline \multirow[t]{2}{*}{ Tavignano } & Amont & Piedicorte & $T 127$ & $\mathrm{~A} 80$ & T132 & A87 & $\begin{array}{l}\text { T110 } \\
\text { T240 }\end{array}$ & A116 & $\mathrm{T} 150$ & $\begin{array}{l}\text { A } 70 \\
\text { A } 172\end{array}$ \\
\hline & Aval & Aléria & T211 & A 190 & T234 & A117 & $\begin{array}{l}\text { M226 } \\
\text { TA366 }\end{array}$ & A 131 & $T 110$ & $\begin{array}{l}\text { A73 } \\
\text { A 171 }\end{array}$ \\
\hline Fium'Orbo & Aval & Ghisonaccia & $\mathrm{T} 100$ & A70 & T157 & A56 & T138 & A53 & $\begin{array}{l}\text { T142 } \\
\text { T280 }\end{array}$ & $\begin{array}{l}\mathrm{A} 30 \\
\mathrm{~A} 64 \\
\mathrm{~A} 400\end{array}$ \\
\hline Gravone & Amont & Tavera & & & & & $\begin{array}{l}\text { T61 } \\
\text { T172 }\end{array}$ & A 1200 & $\begin{array}{l}\text { T50 } \\
\text { T107 }\end{array}$ & A 190 \\
\hline Taravo & Amont & Corrano & & & & & $\begin{array}{l}\text { T38 } \\
\text { T64 }\end{array}$ & $\begin{array}{l}\text { A44 } \\
\text { A104 } \\
\text { A744 }\end{array}$ & $\begin{array}{l}\text { T54 } \\
\text { T107 }\end{array}$ & A285 \\
\hline $\begin{array}{l}\text { Liamone- } \\
\text { Cruzzini }\end{array}$ & Amont & Azzana & & & & & $\begin{array}{l}\text { T52 } \\
\text { T129 }\end{array}$ & $\begin{array}{l}\text { A51 } \\
\text { A } 108\end{array}$ & $\begin{array}{l}\text { T37 } \\
\text { T73 }\end{array}$ & A62 \\
\hline
\end{tabular}

1. Rivière rejoignant le Golo au niveau du barrage de Calacuccia, prélèvements effectués au niveau de Calasima.

\section{a) Les poissons de rivières}

Les figures 2 et 3 montrent les évolutions des concentrations en ${ }^{137} \mathrm{Cs}$ et ${ }^{134} \mathrm{Cs}$ pour, respectivement, les truites et les anguilles du Tavignano (amont et aval) et du Fium'Orbo (aval) pêchées en juillet 1986, 1987, 1988 et 1989. Trois constatations s'imposent, aussi bien pour ${ }^{137} \mathrm{Cs}$ que pour ${ }^{134} \mathrm{Cs}$. En juillet 1986 , les concentrations pour les truites sont significativement plus importantes que celles pour les anguilles dans les trois stations. On obtient les valeurs moyennes suivantes (en Bq.kg. ${ }^{-1}$ frais) :

$$
\begin{array}{lll}
{ }^{137} \mathrm{Cs} & \text { truite } 32,3 & \text { anguille } 7 \\
{ }^{134} \mathrm{Cs} & \text { truite } 15 & \text { anguille } 3,3
\end{array}
$$

Ces moyennes indiquent des rapports ${ }^{137} \mathrm{Cs} /{ }^{134} \mathrm{Cs}$ très voisins de 2,2. 
TABLEAU IV

Espèces prélevées dans les retenues et lacs corses en juillet 1986, 1987, 1988 et 1989 - Poids moyen des lots mesurés ( $\mathrm{g}$ ).

$C$ carpe, CA carassin, $P$ perche, $R$ rotengle, $S$ sandre, $S F$ saumon de fontaine, $T$ truite, TA tanche

\begin{tabular}{|c|c|c|c|c|}
\hline Retenues, lacs & 1986 & 1987 & 1988 & 1989 \\
\hline Teppe Rosse & $\begin{array}{l}\text { C520 } \\
\text { P107 } \\
\text { R104 } \\
\text { S402 }\end{array}$ & $\begin{array}{l}\text { C1200 } \\
\text { P190 } \\
\text { R170 } \\
\text { S225 } \\
\text { TA323 }\end{array}$ & $\begin{array}{l}\text { C 722 } \\
\text { R66 } \\
\text { P 420 } \\
\text { S514 }\end{array}$ & $\begin{array}{l}\text { C800 } \\
\text { C1175 } \\
\text { R240 } \\
\text { P500 } \\
\text { S760 }\end{array}$ \\
\hline Nino & & $\begin{array}{l}\text { T19 } \\
\text { T } 73 \\
\text { T } 74 \\
\text { T } 188\end{array}$ & $\begin{array}{l}\text { T49 } \\
\text { T107 } \\
\text { T191 }\end{array}$ & \\
\hline Rotondo & & $\mathrm{T} 940^{2}$ & & \\
\hline Capitello & & & $\begin{array}{l}\text { SF } 90^{2} \\
\text { SF } 196^{2} \\
\text { SF } 270\end{array}$ & \\
\hline Melo & & & $\begin{array}{l}\text { SF9 } \\
\text { SF40 } \\
\text { SF84 } \\
\text { SF 106 } \\
\text { SF } 171\end{array}$ & \\
\hline Vitalaca & & & & $\begin{array}{l}\text { T86 } \\
\text { T283 }\end{array}$ \\
\hline Bracca & & & & $\begin{array}{l}\text { T160 } \\
\text { T1406 }\end{array}$ \\
\hline Ospedale & $\mathrm{T} 1495^{3}$ & & $\begin{array}{l}\text { C418 } \\
\text { CA132 } \\
\text { R71 } \\
\text { P83 } \\
\text { T66 }\end{array}$ & $\begin{array}{l}\text { C735 } \\
\text { CA167 }\end{array}$ \\
\hline
\end{tabular}

1 Carpe "écailleuse"

2 Eviscérée

3 Eviscérée datant de novembre 1985

Les courbes d'évolution 1986-1989 pour les deux espèces sont différentes ; la diminution des concentrations est beaucoup plus rapide pour la truite que pour l'anguille, surtout durant la première année. Pour l'anguille, il y a même maintien ou légère augmentation de concentration, en 1987, dans les stations Fium'Orbo aval et Tavignano aval ( $\left.{ }^{137} \mathrm{Cs}\right)$. Les 
valeurs moyennes ci-dessous indiquent que celles relatives à l'anguille sont devenues supérieures à celles de la truite, en 1989 (en Bq. gg $^{-1}$ frais). Dans le même temps, le rapport ${ }^{137} \mathrm{Cs} /{ }^{134} \mathrm{Cs}$ est passé à environ 4 pour la truite et à environ 6 pour l'anguille :
${ }^{137} \mathrm{Cs}$
truite 1,15
anguille 2,5
truite 0,3
anguille 0,4

En 1989, les valeurs maximales observées pour la truite sont, pour ${ }^{137} \mathrm{Cs}$ et ${ }^{134} \mathrm{Cs}: 1,5$ et $0,4 \mathrm{~Bq} \cdot \mathrm{kg}^{-1}$ frais ; pour l'anguille, elles sont, respectivement de 3,8 et 0,7 Bq. $\mathrm{kg}^{-1}$ frais.



Fig. 2 - Concentrations en ${ }^{137} \mathrm{Cs}$ et en ${ }^{134} \mathrm{Cs}$ pour les truites du Tavignano et du Fium'Orbo de 1986 a 1989

Nos prélèvements permettent, pour juillet 1988 et juillet 1989, des comparaisons pour l'anguille et pour la truite dans 8 stations situées sur 6 rivières et, en particulier, à l'amont et à l'aval de deux d'entre-elles, le Golo et le Tavignano sur la côte orientale (fig. $4:{ }^{137} \mathrm{Cs}$, anguilles ; fig. $5:{ }^{137} \mathrm{Cs}$, truites; fig. $6:{ }^{134} \mathrm{Cs}$, anguilles et fig. $7:{ }^{134} \mathrm{Cs}$, truites). On retiendra deux aspects importants :

- si l'on ne s'intéresse qu'aux cinq stations amont de ces rivières, il n'apparaît pas de différence significative entre le côte occidentale et la 
côte orientale ; en effet, le rapport des concentrations obtenues est toujours inférieur à 2 ;

- pour le Golo et le Tavignano, on peut considérer que les zones amont sont plus influencées que les zones aval (à l'exception du cas de l'anguille pour le Golo). D'ailleurs, dans le Viru, un petit affluent du Golo ayant sa confluence un peu en amont de Ponte Castirla, on enregistre pour les truites des concentrations en césium plus importantes que celles obtenues à Ponte Castirla.

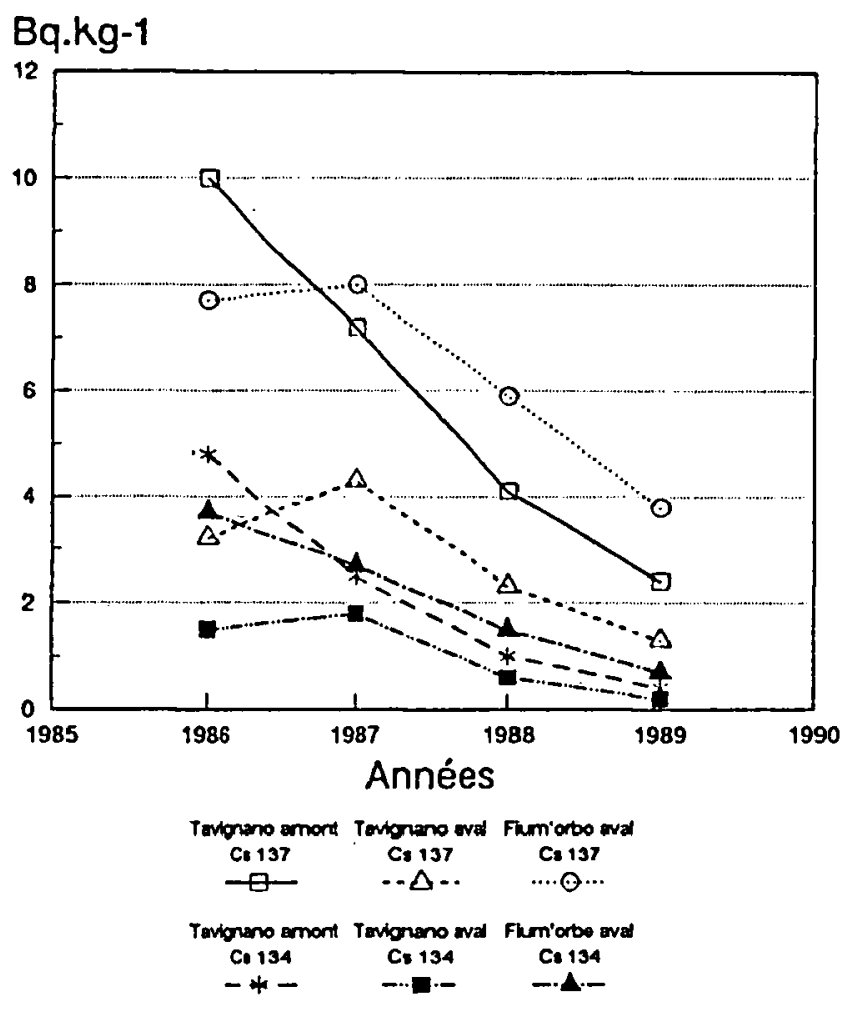

Fig. 3 - Concentrations en ${ }^{137} \mathrm{Cs}$ et en ${ }^{134} \mathrm{Cs}$ pour les anguilles du Tavignano et du Fium'Orbo de 1986 à 1989

\section{b) Les poissons des retenues (fig. 8 et 9)}

Pour les quatre espèces de la retenue de Teppe Rosse et pour les deux radionucléides ${ }^{137} \mathrm{Cs}$ et ${ }^{134} \mathrm{Cs}$, on a deux séries de courbes : celle des espèces carnassières - le sandre et la perche - et celle des espèces omnivores - le rotengle et la carpe. Pour les deux espèces carnassières, les concentrations sont significativement supérieures puisque nous obtenons pour ${ }^{137} \mathrm{Cs}$, en juillet 1986 les valeurs suivantes :
sandre : 178 Bq. $\mathrm{kg}^{-1}$ frais
perche : $82 \mathrm{~Bq} \cdot \mathrm{kg}^{-1}$ frais
rotengle : $21 \mathrm{~Bq} \cdot \mathrm{kg}^{-1}$ frais
carpe : $11 \mathrm{~Bq}^{\mathrm{kg}} \mathrm{kg}^{-1}$ frais 

frais.

Respectivement, les valeurs pour ${ }^{134} \mathrm{Cs}$ sont $84,37,10$ et $5,3 \mathrm{~Bq} \cdot \mathrm{kg}^{-1}$

Une comparaison est possible, en juillet 1988, avec la retenue de l'Ospedale située plus au sud, à l'ouest de Porto Vecchio. Pour les trois espèces communes aux deux retenues, les valeurs pour l'Ospedale sont toujours supérieures. Pour ${ }^{137} \mathrm{Cs}$, on obtient les couples de valeurs suivantes (en Bq. $\mathrm{kg}^{-1}$ frais) :

$$
\begin{aligned}
& \text { carpe }: 5,4 \text { et } 1,1 \\
& \text { rotengle }: 5 \text { et } 1,1 \\
& \text { perche }: 9,2 \text { et } 8,1
\end{aligned}
$$

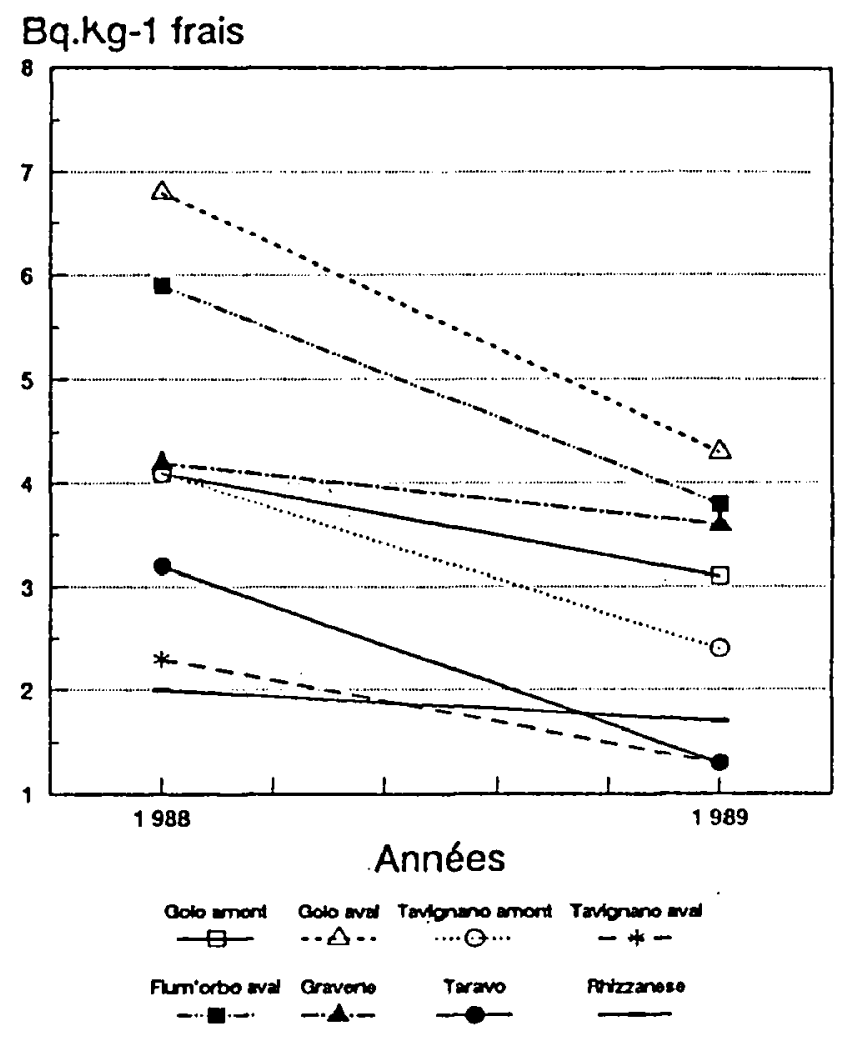

Fig. 4 - Concentrations en ${ }^{137}$ Cs pour les anguilles dans 6 rivières (8 stations) corses en 1988 et 1989 


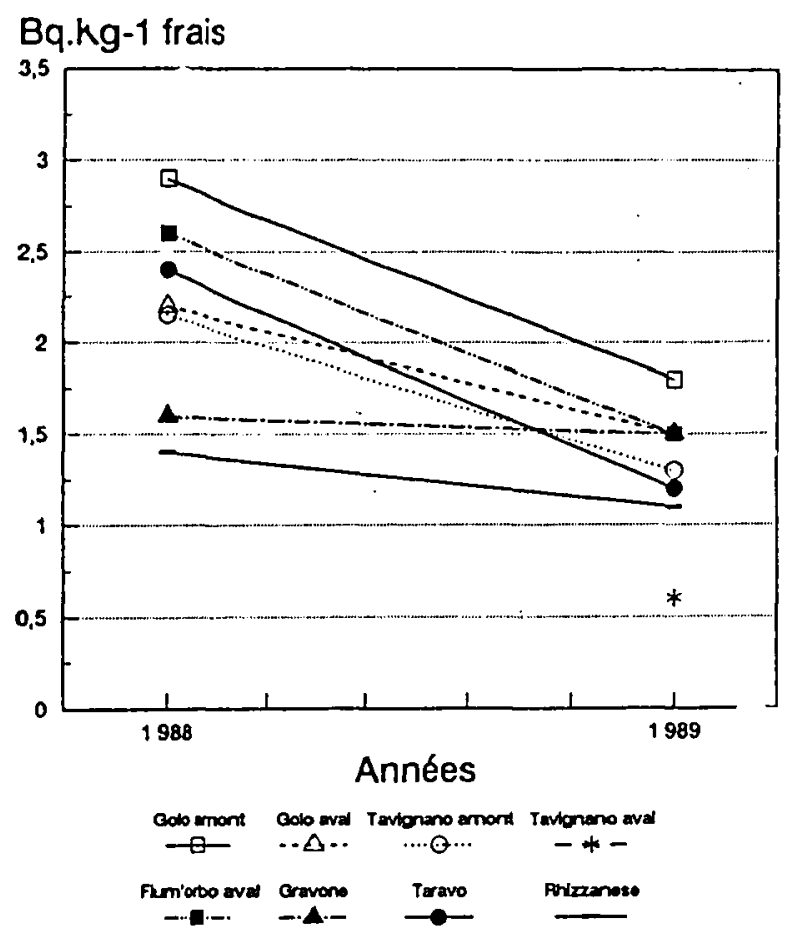

Fig. 5 - Concentrations en ${ }^{137}$ Cs pour les truites dans 6 rivières (8 stations) corses en 1988 et 1989

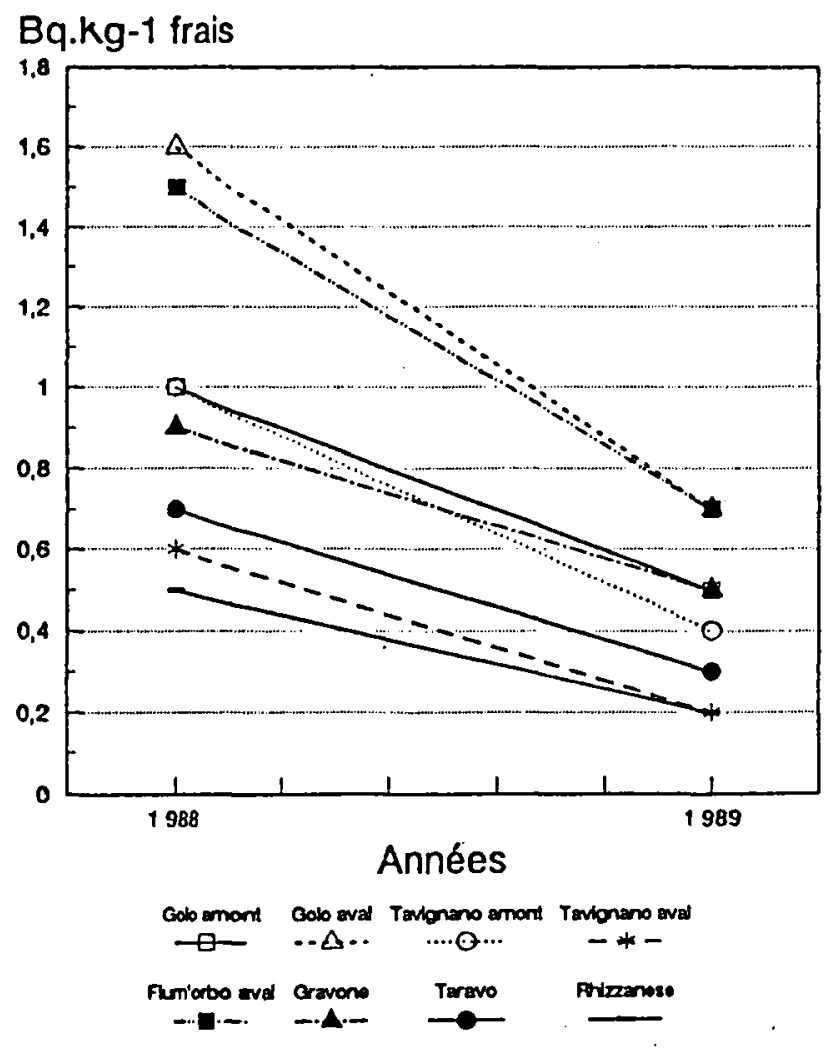

Fig. 6 - Concentrations en ${ }^{134}$ Cs pour les anguilles dans 6 rivières (8 stations) corses en 1988 et 1989 
IMPACT DES RETOMBÉES DE TCHERNOBYL SUR LES POISSONS DE CORSE

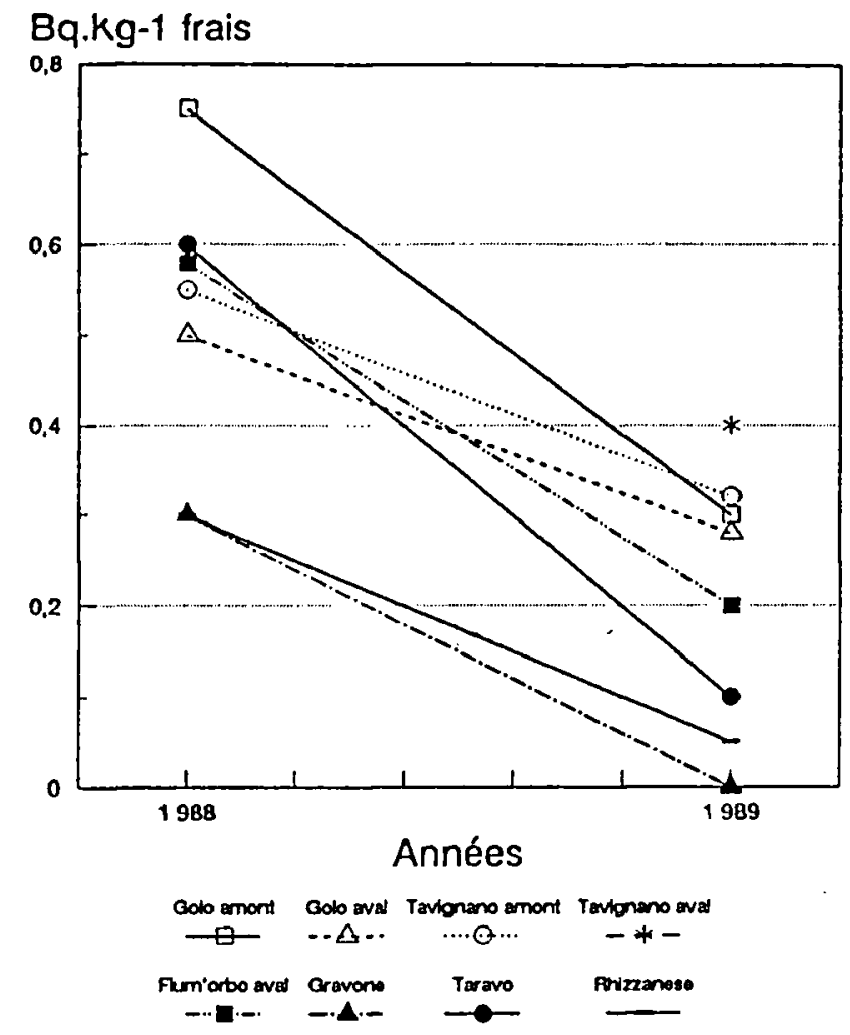

Fig. 7 - Concentrations en ${ }^{134}$ Cs pour les truites dans 6 rivières (8 stations) corses en 1988 et 1989



Fig. 8 - Concentrations en ${ }^{137}$ Cs dans les poissons de la retenue de Teppe Rosse de 1986 à 1989 
B. DESCAMPS

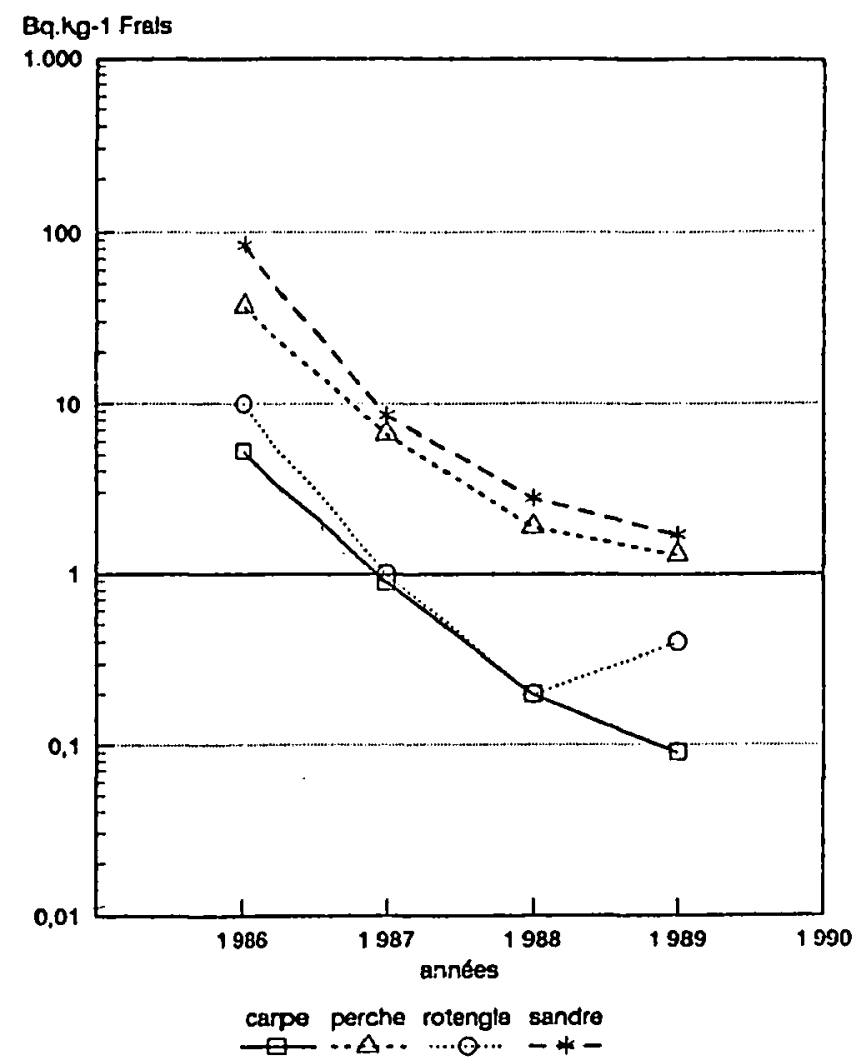

Fig. 9 - Concentrations en ${ }^{134}$ Cs dans les poissons de la retenue de Teppe Rosse de 1986 à 1989



Fig. 10 - Concentrations en ${ }^{137} \mathrm{Cs}$ et ${ }^{134} \mathrm{Cs}$ pour des truites du lac de Nino en 1987 et 1988 


\section{c) Les poissons des lacs de montagne}

Pour le lac de Nino, l'évolution 1987-1988 (fig. 10) peut être comparée à celle des truites du Tavignano et du Fium'Orbo pour la même période (fig. 2). On constate que les valeurs de 1987 pour ${ }^{137} \mathrm{Cs}$ sont comparables (de l'ordre de $10 \mathrm{~Bq} \cdot \mathrm{kg}^{-1}$ frais contre 2,5). On en conclut que la diminution est un peu plus importante en rivières que dans les lacs. En corollaire, on peut alors supposer que, dans ce lac, les valeurs de 1986 étaient supérieures à celle obtenues en rivières, c'est-à-dire 32,3 Bq. $\mathrm{kg}^{-1}$ frais pour ${ }^{137} \mathrm{Cs}$ et 15 pour ${ }^{134} \mathrm{Cs}$.

Pour le lac Capitello situé au-dessus du lac Melo, d'une part, et pour les lacs Bracca et Vitalaca situés dans la même région, d'autre part, il apparaît que les concentrations obtenues pour le saumon de fontaine (Capitello et Melo) et pour la truite (Bracca et Viralaca) sont influencées par l'altitude (fig. 11). On notera que cette influence semble plus importante pour le couple Melo-Capitello que pour le couple Vitalaca-Bracca. Cette influence de l'altitude, non homogène selon les zones concernées, est mise en évidence quand on extrapole la décroissance obtenue sur le lac Nino entre 1987 et 1988 aux autres lacs. En effet, en supposant cette décroissance (fig. 10) valable en durée et en intensité depuis l'accident jusqu'à 1989, on obtient, pour juillet 1986, le classement suivant, par ordre décroissant de contamination :

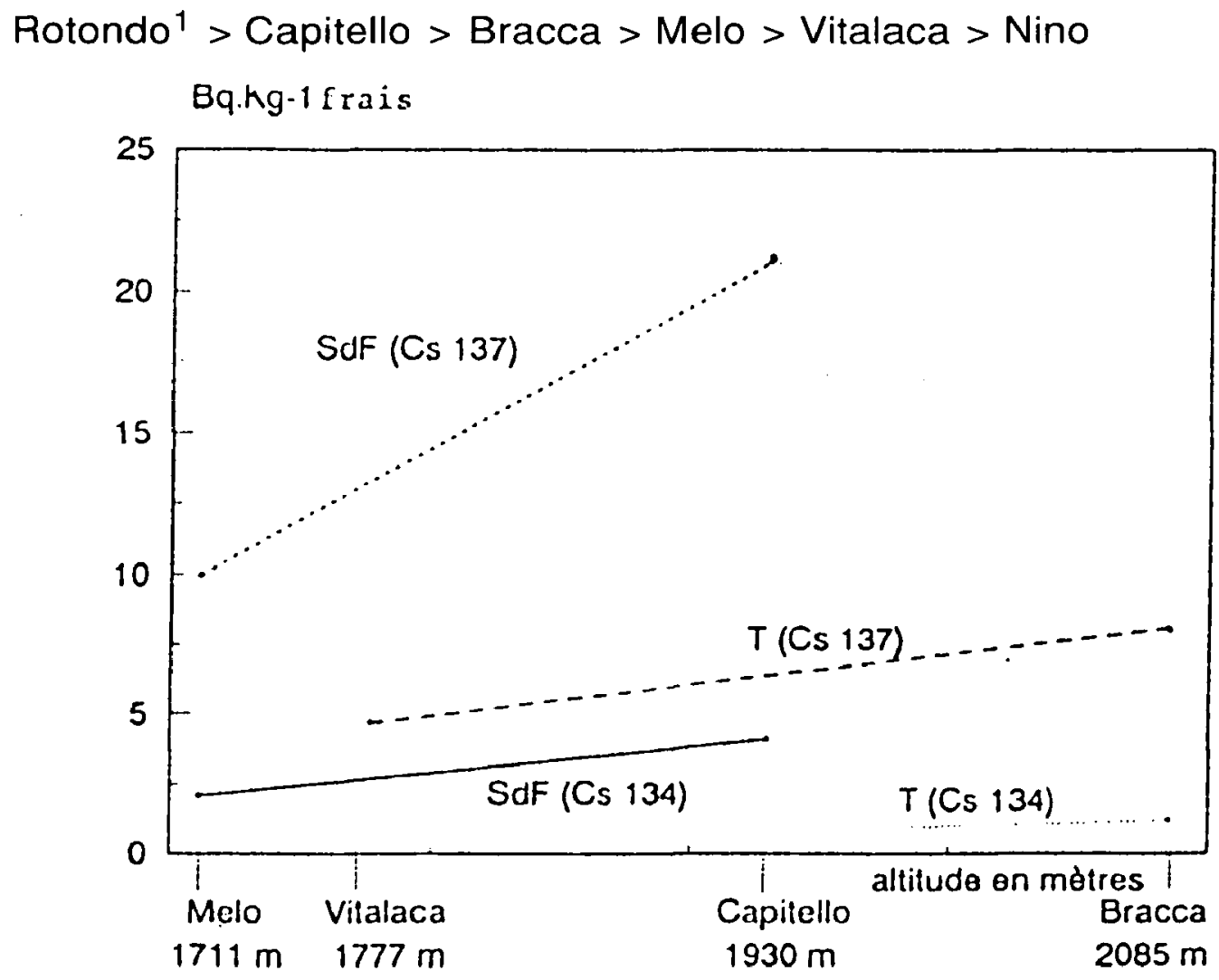

Fig. 11 - Concentrations en ${ }^{137} \mathrm{Cs}$ et ${ }^{134} \mathrm{Cs}$ pour des saumons de fontaine (SdF) dans les lacs de Melo et Capitello en 1988 et pour des truites (T) dans les lacs de Vitalaca et Bracca en 1989, en fonction de l'altitude

1. En juillet 1987 , on a obtenu la valeur de $32 \mathrm{~Bq} \cdot \mathrm{kg}^{-1}$ frais pour la truite $\left({ }^{137} \mathrm{Cs}\right)$ 


\section{d) Résultats relatifs aux autres radionucléides}

En $1986,{ }^{110 \mathrm{~m}} \mathrm{Ag}$ a été décelé dans presque tous les prélèvements : dans les truites et les anguilles des deux stations du Tavignano et dans la station du Fium'Orbo, dans les carpes et les rotengles du Teppe Rosse. Pour les truites, les valeurs s'échelonnent entre 1,5 et $2,1 \mathrm{~Bq} \cdot \mathrm{kg}^{-1}$ frais alors que pour les anguilles elles sont, là encore, plus faibles - entre 0,1 et 0,3 . Les deux valeurs significatives dans la Teppe Rosse sont 1,9 et $0,9 \mathrm{~Bq} \cdot \mathrm{kg}^{-1}$ frais. En 1987, ce radionucléide n'est plus présent que dans cinq échantillons sur quinze et plus particulièrement dans les truites pêchées dans les lacs Rotondo (2,7 Bq. $\mathrm{kg}^{-1}$ frais) et Nino $\left(0,5\right.$ Bq. $\mathrm{kg}^{-1}$ frais $)$.

Nous avons également décelé, en $1986,{ }^{106} \mathrm{Ru}+\mathrm{Rh}$ dans les carpes du Teppe Rosse (4,6 Bq. $\mathrm{kg}^{-1}$ frais) et ${ }^{103} \mathrm{Ru}$, encore dans ces carpes (2,6 Bq. $\mathrm{kg}^{-1}$ frais), mais aussi dans les anguilles de la station de Tavignane amont $0,9 \mathrm{~Bq} \cdot \mathrm{kg}^{-1}$ frais).

\section{e) Résultats relatifs aux unios}

Comme les poissons, les unios prélevés à la station Tavignano aval et mesurés par leurs parties molles, révèlent la présence de ${ }^{137} \mathrm{Cs}$ et de ${ }^{134} \mathrm{Cs}$ en $1986,1987,1988$ et 1989 et celle de ${ }^{103} \mathrm{Ru}$ et de ${ }^{106} \mathrm{Ru}+\mathrm{Rh}$ en 1986 seulement (tableau V). Les ordres de grandeur n'étant pas très importants (maximum de 5,4 Bq. $\mathrm{kg}^{-1} \mathrm{sec}$ en 1986 pour ${ }^{137} \mathrm{Cs}$ ), ces résultats confirment ce que nous avons toujours personnellement pensé : ces lamellibranches d'eau douce ne sont pas des radioindicateurs particulièrement intéressants.

Tableau $\mathrm{V}$

Concentrations obtenues pour des Unios prélevés en Corse en juillet 1986, 1987, 1988 et 1989 dans le Tavignano à la station Aléria. Valeurs pour les parties molles en Bq. $\mathrm{kg}^{-1}$ sec

\begin{tabular}{|cccccc|}
\hline Années & ${ }^{137} \mathbf{C s}$ & ${ }^{134} \mathbf{C s}$ & $\begin{array}{c}\text { Radionucléides } \\
\mathbf{1 0 6} \mathbf{R u}+\mathbf{R h}\end{array}$ & ${ }^{103} \mathbf{R u}$ & ${ }^{137} \mathbf{C s} /{ }^{134} \mathbf{C s}$ \\
\hline 1986 & $5,4 \pm 0,9$ & $2,5 \pm 0,3$ & $13 \pm 2,2$ & $12 \pm 1,1$ & 2,2 \\
1987 & $1,4 \pm 0,2$ & $0,5 \pm 0,02$ & - & - & 2,8 \\
1988 & $1,2 \pm 0,2$ & $0,3 \pm 0,1$ & - & - & 4 \\
1989 & $1,5 \pm 0,2$ & $0,2 \pm 0,1$ & - & - & 7,5 \\
& $0,7 \pm 0,3^{1}$ & - & - & - & - \\
\hline
\end{tabular}

1 Coquilles 


\section{Comparaison avec d'autres régions françaises et européennes et discussion}

Nous pouvons comparer ces résultats de Corse (de 1986 et 1987) avec ceux obtenus par nous-même dans deux autres régions françaises reconnues, dès mai 1986, avoir subi l'influence des retombées : le bassin du Var dans le sud-est et la partie en amont de la centrale nucléaire de Cattenom (donc hors de son influence), sur la Moselle [2]. Globalement les résultats de Corse sont confirmés.

Dans le bassin du Var et ses affluents - l'Estéron, la Vésubie et la Tinée - on peut effectuer des comparaisons sur l'anguille et la truite. II y a confirmation sur deux points $\left({ }^{137} \mathrm{Cs}\right.$ et $\left.{ }^{134} \mathrm{Cs}\right)$ :

- en octobre 1986 les concentrations pour la truite sont plus importantes que pour l'anguille, truite.

- l'évolution (diminution) 1986-1987 est beaucoup plus rapide chez la

On notera, cependant, que les niveaux d'activité dans le bassin du Var sont un peu plus faibles qu'en Corse (d'un facteur 2 à 3 ).

En Moselle, là où le peuplement est beaucoup plus diversifié, nous avons encore des confirmations. On peut les résumer, en les affinant toutefois, par la mise en valeur de trois catégories de poissons:

- ceux qui ont atteint leur concentration maximale dès le 15 mai 1986 (chevaine, rotengle et probablement gardon),

- ceux qui l'ont atteinte en octobre ou novembre 1986 (anguille et brême),

- ceux qui ont vu ce phénomène amplifié (brocher et perche).

Avec la réserve d'usage concernant la difficulté de comparer des mêmes espèces vivant dans des milieux différents (perche et rotengle en Moselle et dans la retenue de Teppe Rosse en Corse), il apparaît que la Moselle a été un peu moins influencée que la Corse.

Pour le territoire français, on ne serait pas complet sans fournir ces deux éléments :

- en Corse, la seule valeur en notre possession pour la période avant Tchernobyl concerne la truite prélevée en novembre 1985 dans la retenue de l'Ospedale ( ${ }^{137} \mathrm{Cs}: 2,1 \mathrm{~Bq} \cdot \mathrm{kg}^{-1}$ frais),

- dans les rivières françaises, hors d'influence d'installations nucléaires civiles, seul ${ }^{137} \mathrm{Cs}$ est présent, les niveaux de concentrations étant compris entre 0,05 et $0,5 \mathrm{~Bq} \cdot \mathrm{kg}^{-1}$ frais [4].

Pour ce qui concerne les poissons de rivières et de lacs du reste de l'Europe, on dispose d'une très récente synthèse bibliographique exhaustive, commandée par la Commission des communautés européennes (CCE) et par l'Union internationale des radioécologistes (UIR) [3]. Nous 
allons en ressortir les principaux aspects (pour les poissons) en les confrontant avec nos résultats.

En tout premier lieu, il y a confirmation de la prédominance des deux radiocésium, puis de ${ }^{103} \mathrm{Ru}+{ }^{106} \mathrm{Rh}$ et ${ }^{110 \mathrm{~m}} \mathrm{Ag}$.

Localement, il est clairement démontré que les variations observées sont dues à l'influence des courants atmosphériques, de la distance au point d'émission et de la pluviométrie. Ce sont donc les dépôts humides locaux qui représentent le paramètre essentiel des différents impacts régionaux.

Pour ce qui concerne les poissons de rivière, les concentrations s'échelonnent entre quelques Bq. $\mathrm{kg}^{-1}$ frais et 400 (en Suède) ; généralement, les concentrations les plus élevées sont obtenues pour la perche, le brochet et parfois la truite. Les valeurs maximales enregistrées en Corse en juillet 1986 (de l'ordre de $40 \mathrm{~Bq} \cdot \mathrm{kg}^{-1}$ frais pour la truite) se situent dans la zone intermédiaire entre les minimums $\left(<20 \mathrm{~Bq} \cdot \mathrm{kg}^{-1}\right.$ frais en Autriche et en Rhénanie-Palatinat) et les maximums. La constatation d'une très faible (ou d'une absence de) différence entre la côte orientale et la côte occidentale implique des pluviométries comparables sur les deux versants de la chaîne centrale et la présence d'un panache local d'altitude élevé (> $2500 \mathrm{~m}$ ).

Pour les poissons lacustres, les variations locales sont beaucoup plus importantes. Des concentrations en ${ }^{137} \mathrm{Cs}$ de plusieurs milliers de Bq. $\mathrm{kg}^{-1}$ frais sont assez courantes dans les lacs suédois et finlandais. Là encore, les carnassiers présentent les valeurs les plus élevées. C'est ce que nous obtenons dans les deux retenues de Corse (sandre et perche) mais avec au moins une décade de différence (maximum $178 \mathrm{~Bq} \cdot \mathrm{kg}^{-1}$ frais).

Nos résultats sont en opposition avec les données de cette synthèse bibliographique sur au moins un aspect : la durée de la période biologique effective "apparente"1 en lacs et en rivières. En effet, cette synthèse met en exergue une beaucoup plus longue période biologique pour les poissons de lac par rapport aux poissons de rivière. En Corse, les périodes biologiques courtes pour les différentes espèces de la retenue de Teppe Rosse s'échelonnent entre 77 jours pour le sandre et 115 jours pour la perche tandis que pour la truite, en rivière, la période courte est 138 jours et la longue 231. De plus, toujours en rivière, la valeur pour l'anguille est voisine de 800 jours.

\section{Impact sanitaire potentiel de la consommation de poissons}

II y a en Corse 30 associations de pêche et de pisciculture (APP) regroupant 6500 pêcheurs, tous les cours d'eau étant en première caté-

\footnotetext{
- Il faut ici utiliser - comme tous les auteurs le font en présence de données de terrain le qualificatif "apparente" car, en toute logique, il y a encore eu après le premier impact initial important, des apports pour les poissons. Aussi bien par la voie eau directe (drainage retardé des bassins versants par exemple) que par la voie trophique.
} 
gorie (pêche à la truite). Il est donc légitime de s'intéresser à l'impact qu'a eu ou qu'aurait pu avoir la consommation de poissons. Dans ce chapitre, nous évaluons l'équivalent de dose efficace après l'ingestion annuelle de $10 \mathrm{~kg}$ de poissons. On considérera, pour ${ }^{137} \mathrm{Cs}$ et ${ }^{134} \mathrm{Cs}$, que les concentrations obtenues pour l'organisme entier sont les mêmes (et c'est généralement le cas) que celles des parties consommables (les muscles). Trois cas sont envisagés, en 1986 et 1989 :

- le sandre de Teppe Rosse pour qui on a obtenu les valeurs maximales,

- la truite de la station Fium'Orbo aval,

- l'anguille de la station Tavignano amont.

Les éléments de calcul à prendre en compte sont :

$\mathrm{D}=\mathrm{Q} . \mathrm{C} . \mathrm{k}$

où : $\quad$ D est l'équivalent de dose efficace, en Sv. $a n^{-1}$

$Q$ est la consommation annuelle de poisson, en $\mathrm{kg}$

Bq. $\mathrm{kg}^{-1}$ frais

$\mathrm{C}$ est la concentration en ${ }^{137} \mathrm{Cs}$ et en ${ }^{134} \mathrm{Cs}$ du poisson, en

$\mathrm{k}$ est le facteur de dose, en Sv.Bq-1

pour ${ }^{137} \mathrm{Cs}$, $k$ est égal à $1,4 \times 10^{-8}$

pour ${ }^{134} \mathrm{Cs}$, k est égal à $2 \times 10^{-8}$.

Le tableau $\mathrm{VI}$ indique les valeurs de $\mathrm{D}$ et la proportion qu'elles représentent par rapport à la dose population $\left(5 \mathrm{mSv} . \mathrm{an}^{-1}\right)$ bien que celle-ci soit applicable aux situations normales à caractère chronique et puisse être subie par un individu chaque année toute sa vie durant.

TABLEAU VI

Equivalent de dose efficace correspondant

à l'ingestion annuelle de $10 \mathrm{~kg}$ de poissons de Corse influencés par les retombées de Tchernobyl

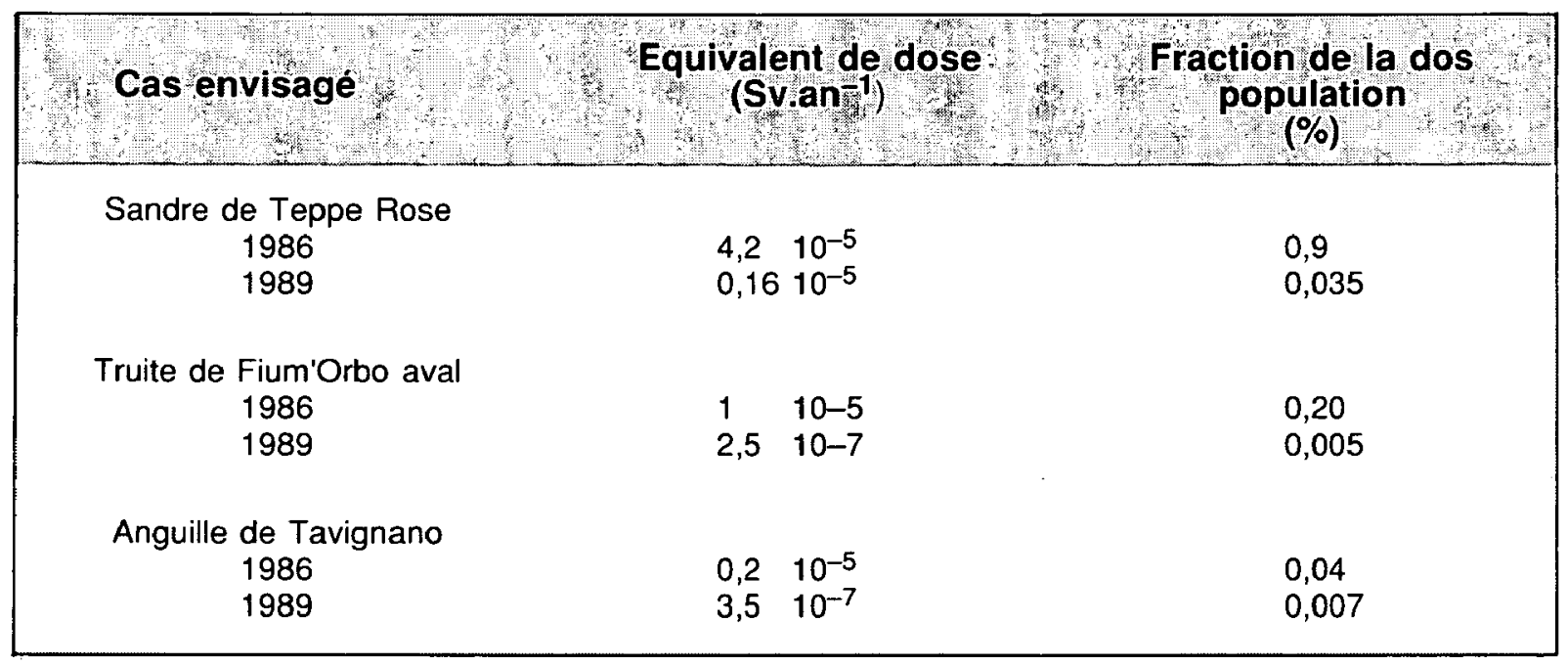

1. Coquilles 


\section{CONCLUSION}

L'étude de l'incidence des retombées de Tchernobyl sur les poissons des six principales rivières corses -3 de la façade orientale et 3 la façade occidentale - de deux retenues artificielles et de six lacs de haute montagne a été réalisée en juillet 1986, 1987, 1988 et 1989.

On s'est intéressé au peuplement dominant : truite (ou saumon de fontaine) et anguille dans les rivières et les lacs de haute montagne ; sandre, perche, carpe, carassin et rotengle dans les retenues artificielles.

L'incidence de Tchernobyl est caractérisée, en juillet 1986, par la présence de ${ }^{137} \mathrm{Cs}$ et de ${ }^{134} \mathrm{Cs}$; elle est encore effective bien que très atté-

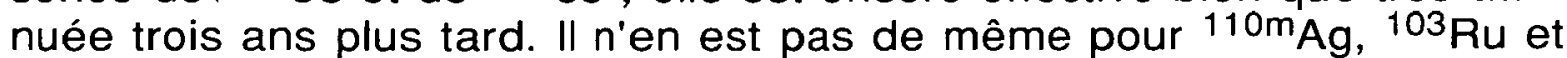
${ }^{106} \mathrm{Ru}+\mathrm{Rh}$ qui, quand ils étaient présents, ont presque toujours disparu un an après l'accident.

En rivière, la truite est plus affectée que l'anguille, en juillet 1986 ; trois ans après, c'est l'inverse. On a pu d'ailleurs calculer que la période biologique de l'anguille est voisine de 2 ans (on ne peut en calculer qu'une seule) alors que celles de la truite sont de 138 jours (Tb1) et 231 jours (Tb2).

En retenue artificielle, les espèces carnassières sont significativement plus contaminées que les espèces dites "omnivores" (en juillet 1986) ; les décroissances sont, par contre, relativement comparables puisque les périodes biologiques courtes sont comprises entre 77 et 115 jours.

Dans les six lacs de haute montagne (entre $1710 \mathrm{~m}$ et $2321 \mathrm{~m}$ ), on a pu montrer une corrélation positive entre les concentrations dans les salmonidés présents et l'altitude.

On retiendra également que l'influence sur la façade occidentale est à peine inférieure à celle de la façade orientale.

En terme d'impact sanitaire, le cas le plus défavorable (sandre de la retenue de Teppe Rosse en juillet 1986) ne pouvait induire que 0,9\% de la dose population autorisée. En 1989, ce chiffre est divisé par un facteur 25. Pour cette même année, la consommation de truite, la plus probable compte tenu de la recherche des pêcheurs, ne représenterait que $0,005 \%$ de la dose population.

\section{REMERCIEMENTS}

Cette étude n'aurait pas été possible sans l'amitié et le concours de MM. CRISTOFARI, MATTEI et FILIPPI, les trois garde-pêches de Bastia. Ce texte leur est dédié.

Mes remerciements vont à tous ceux qui ont collaboré, ou favorisé la collaboration, au cours des quatre campagnes de prélèvements. Je pense là tout particulièrement à $M$. ROCHE du SRAE de Bastia, MM. RIVIER et DUMONT du CEMAGREF d'Aix-en-Provence, M. LENK du 
PNRC, Mme BAUDIN-JAULENT et M. REMILLET du Laboratoire de radioécologie des eaux continentales de Cadarache.

Les mesures de spectrométrie $\gamma$ ont été réalisées par le LMEI d'Orsay; je profite de cette occasion pour rendre hommage à M. HEMON, récemment disparu.

\section{BIBLIOGRAPHIE}

[1] COMMISSARIAT A L'ÉNERGIE ATOMIQUE. INSTITUT DE PROTECTION ET SURETÉ NUCLÉAIRE (IPSN). L'accident de Techernobyl, Rapport IPSN 2/86 révision 3. Fontenay aux Roses: IPSN, 1986, 163 pages.

[2] DESCAMPS B. \& BAUDIN-JAULENT Y. Evolution de l'impact radioécologique de l'accident de Tchernobyl dans trois bassins fluviaux français. In : Impact des accidents d'origne nucléaire sur l'environnement, IVème Symposium international de radioécologie, Cadarache, France, 14-18 mars 1988. Cadarache : CEA, DERSSERE, 1988, C68-680.

[3] FOULQUIER L. \& BAUDIN-JAULENT Y. (1989). Impact radioécologique de l'accident de Tchernobyl sur les écosystèmes aquatiques continentaux. CCE (Radioprotection, 50 Rapport XI - 3522/90FR) : Luxembourg : CCE, 1989, 216 pages.

[4] LAMBRECHTS A., FOULQUIER L. \& PALLY M. (1988). Etude comparée de l'impact radioécologique des installations nucléaires et de l'accident de Tchernobyl sur le fleuve Rhône. In : Impact des accidents d'origine nucléaire sur l'environnement. IVème symposium international de radioécologie, Cadarache, 14-18 mars 1988. Cadarache : CEA, DERS-SERE, 1988, vol. 1, C39-C50.

[5] LEGLIZE L. \& CROCHARD C. Vérification expérimentale du choix de Dreissena polymorpha PALLAS (lamellibranche) comme bioindicateur de contamination métallique. Nat Can. (Rev. Ecol. Syst.), 1987, 114, 315-323.

[6] RIVIER B. \& DUMONT B. Etude ichtyologique des lacs d'altitude corses. II - Le lac de Rotondo. III - Le lac de Nino. Rapport CEMAGREF-PNRC, juin 1988, 91 pages. 